\title{
A Successful Treatment of Chronic Migraine with Hyperbaric Oxygen Therapy
}

\author{
Authors: \\ Rahmah Shafee, ${ }^{1}$ Mohd Amirullah Hamzah, ${ }^{1}$ Rajesh Kumar Muniandy² \\ 1. Healing Chambers of Asia, Kuala Lumpur, Malaysia \\ 2. Faculty of Medicine and Health Sciences, Universiti Malaysia Sabah, Kota Kinabalu, \\ Malaysia \\ *Correspondence to rajeshkumar.ums@gmail.com
}

Disclosure: The authors have declared no conflicts of interest.

Received: $\quad 03.11 .20$

Accepted: $\quad 08.01 .21$

Keywords: $\quad$ Alternative treatment, hyperbaric oxygen therapy (HBOT), migraine.

Citation: $\quad$ EMJ Neurol. 2021; DOI/10.33590/emjneurol/20-00262

\begin{abstract}
The World Health Organization (WHO) ranks migraine as the most prevalent and disabling neurological condition. Management of migraines can be broadly divided into lifestyle and trigger management, acute treatments, and preventive treatments. Despite pharmacological advances, this medical problem has remained undertreated.

A 40-year-old male presented to the authors' hospital and complained of severe, constant, and throbbing pain over the right side of his head. There was occasional photophobia but no visual disturbances. Oral analgesics were not helpful. After careful evaluation, he was advised to receive hyperbaric oxygen therapy sessions. He underwent one session per day, 5 days per week for 4 weeks. At the end of the 20 sessions, the patient reported an improvement in his symptoms, and did not require any oral medication. The authors concluded that hyperbaric oxygen therapy should be explored further as a treatment for pharmacologically resistant migraine.
\end{abstract}

\section{INTRODUCTION}

Migraines involve intense head pain, which severely affects the activities of daily living, and is the is the third most common disease in the world. ${ }^{1}$ Migraines affect approximately $15 \%$ of the general population in England ${ }^{2}$ and the World Health Organization (WHO) ranks migraine as the most prevalent and disabling neurological condition. ${ }^{3}$ Patients with migraine usually complain of pain over half of the head. The pain is generally throbbing in nature and worsens with movement. It is typically accompanied by nausea, dizziness, extreme sensitivity to lights and noises, lack of appetite, disturbances of bowel function, and occasionally aura. ${ }^{4}$ There are four phases of migraine, ${ }^{5}$ which have been summarised in Table 1.

\section{PATHOGENESIS}

The exact pathogenesis of migraine is unknown. The current state of knowledge suggests that a primary neuronal dysfunction leads to a sequence of changes intracranially and extracranially. 6,7 This primary neuronal dysfunction has features of cortical spreading depression, activation of the trigeminovascular system, onset of inflammation, and sensitisation. 


\begin{tabular}{|l|l|l|}
\hline Phases & Time period & Symptoms \\
\hline Prodome & A few days to hours & $\begin{array}{l}\text { Irritability, depression, yawning, increased } \\
\text { need for urination, food craving, sensitivity } \\
\text { to light and sounds, difficulty concentrating, } \\
\text { fatigue, difficulty speaking/reading, nausea, } \\
\text { difficulty sleeping }\end{array}$ \\
\hline Aura & 5-60 min & $\begin{array}{l}\text { Visual disturbances, temporary loss of sight, } \\
\text { numbness and tingling on part of the body }\end{array}$ \\
\hline Migraine attack & $4-72$ hours & $\begin{array}{l}\text { Throbbing pain, burning sensation, nausea, } \\
\text { vomiting, giddiness, insomnia, nasal congestion, } \\
\text { anxiety, depressed mood, sensitivity to light/ } \\
\text { smell/sound, neck pain and stiffness }\end{array}$ \\
\hline Postdrome & 24-48 hours & $\begin{array}{l}\text { Inability to concentrate, fatigue, depressed } \\
\text { mood, euphoric mood, lack of comprehension }\end{array}$ \\
\hline
\end{tabular}

The once-popular vascular theory of migraine, which suggested that migraine headache was caused by the dilatation of blood vessels, while the aura of migraine resulted from vasoconstriction, is no longer considered viable..$^{8-10}$ Vasodilatation, if it occurs at all during spontaneous migraine attacks, ${ }^{10}$ is probably an epiphenomenon resulting from instability in the central neurovascular control mechanism.

Currently considered to be a neurological condition, migraine is more likely caused by inflammation in response to the release of neuropeptides. The release of neuropeptides, such as Substance $P$, calcitonin gene-related peptide, and neurokinin $A$, is associated with the process of neurogenic inflammation, which is thought to be important in the prolongation and intensification of the pain of migraine." This neurogenic inflammation can lead to sensitisation: a process in which neurons become increasingly responsive to stimulation and the pain threshold decreases. Sensitisation is thought to be responsible for many of the clinical symptoms of migraine, including the throbbing quality of the pain and worsening of the pain with coughing, bending, or sudden head movements. ${ }^{12-14}$

Management of migraines can be broadly divided into three: lifestyle and trigger management, acute treatments, and preventive treatments. Some examples of the acute treatment for migraine are paracetamol, nonsteroidal antiinflammatory drugs, opioids, and triptans. Tricyclics, $\beta$-blockers, anticonvulsants, and botulinum toxin (botox) injections are some options for preventive treatments; however, despite advances in pharmacology, migraine has remained undertreated. ${ }^{4}$ Besides pharmacological treatment, there are other modalities that are emerging to be treatment options to treat migraine. Among these modalities, hyperbaric oxygen therapy (HBOT) is gaining popularity. The authors present a case of a patient with chronic migraine, managed successfully with HBOT.

\section{CASE DESCRIPTION}

A 40-year-old male patient complained of frequent severe, constant, and throbbing pain over the right side of his head. There was occasional photophobia but no visual disturbances. He had a history of migraine for $>10$ years. Initially, the attacks occurred two to three times per month, but had increased to more than 15 times per month in the preceding 6 months. Each pain episode would last for 1-2 hours. The patient's Numerical Rating Scale (NRS) pain score during the attack had also increased over the years, from 6/10 to 10/10. Over the years, he had a history of taking oral sumatriptan, nonsteroidal anti-inflammatory drugs, antiepileptics, and opioids. None of the pharmacotherapy helped to reduce his pain or frequency of attacks. 
Upon examination, the patient was alert and orientated. His vital signs were within normal range. His motor and sensory examinations and reflexes for both upper and lower limbs were healthy. Examination of his cranial nerves was also normal. There were no visible lesions on his face or eye and brain MRI was normal. Based on the International Classification of Headache Disorders $3^{\text {rd }}$ edition (ICHD-3) guidelines, a diagnosis of chronic migraine was made. The patient presented to the authors' clinic requesting for an alternative treatment for his migraine. He was unhappy with his existing pain control. After careful evaluation, he was advised to receive 20 sessions of 90-minute HBOT at 2 atmosphere absolute (ATA). The plan was to administer one session per day, 5 days per week, for a duration of 4 weeks.

A single-person-chamber was used for the treatment. During the treatment in the chamber, the patient did not have any discomfort or complaints and was comfortable in the chamber. No unwanted effects of HBOT were recorded following sessions. At the end of the 20 sessions, the patient reported an improvement in his symptoms. His pain score reduced to $2 / 10$. He did not require analgesics or any prophylactic medication. He was able to continue with his daily activities and work without any complaints. A follow-up review of the patient 6 months later showed good pain control, with a pain score ranging from 1 to 3 during his migraine attacks. The frequencies of attack were also reduced to one or two episodes per month. He did not require any analgesics or any more HBOT maintenance.

\section{DISCUSSION}

The Undersea and Hyperbaric Medical Society (UHMS) defined HBOT as an intervention in which an individual breathes near 100\% oxygen intermittently while inside a hyperbaric chamber that is pressurised to greater than sea level pressure ( 1 ATA $)^{15}$ If the lungs are healthy, an arterial partial pressure of oxygen in the range $1000-15000 \mathrm{mmHg}$ is generally achieved at 2-3 ATA. This will create an oxygen partial pressure in the tissues of between 200 and $400 \mathrm{mmHg}$ or more, which will induce a controlled oxidative stress causing activation of various pathways. This will result in multiple potential clinical benefits, including diminished inflammatory responses. ${ }^{16}$ In a monoplace chamber, an individual patient breathes in directly pressurised 100\% oxygen. In the multiplace chambers with more than one patient, the patients will be pressurised with air, and breathe $100 \%$ oxygen indirectly via a head hood, mask, or endotracheal tube. ${ }^{17}$ HBOT is approved for 14 indications, summarised in Table $2 ;{ }^{15}$ however, besides these standard indications, several studies have demonstrated many other benefits of HBOT, including the management of migraines.

There have been several published studies regarding the use of $\mathrm{HBOT}$ to treat migraine. There was a similar case report recently showing a successful treatment of migraine in a 23-yearold patient. ${ }^{18}$ The authors used a treatment of 1.5 ATA in a chamber for 40 sessions. This protocol will reduce the treatment time by $50 \%$, as the authors only held 20 sessions with the patient instead of the documented 40 sessions. The 20-session intervention protocol had the added advantage of less disruption to the patient's routine and productivity as well as faster positive outcome. Despite a higher ATA, the patient was comfortable without any complaints. This protocol had a good outcome in the patient. The authors will continue to use this treatment protocol for future patients with severe migraine.

Oxygen is presumed to have the ability to constrict distal cerebral vessels; ${ }^{19}$ therefore, oxygen administered at higher pressures will produce even further vasoconstriction. The supply of oxygen dissolved within the plasma, however, is not compromised and the net effect of tissue oxygen is maintained because of hyperoxia. ${ }^{20}$ This suggests that HBOT may be a favourable treatment for vascular migraines. ${ }^{21}$ Furthermore, the pain relief effects of HBOT for those with migraine may also be attributed to the correction of local hypoxia.

If the current projections about the pathophysiology of migraine are correct, inflammation plays a central role, and vasodilatation is an epiphenomenon. Hyperbaric oxygen has been shown to modulate the immune cytokines in multiple conditions, including decompression illness and air embolism; that would be the likely mechanism of benefit in patients with migraine. ${ }^{22}$ 
Table 2: Indications for hyperbaric oxygen therapy.

\begin{tabular}{|l|}
\hline Air or gas embolism \\
\hline Carbon monoxide poisoning \\
\hline Gas gangrene \\
\hline Crush injury, compartment syndrome \\
\hline Decompression sickness \\
\hline Arterial insufficiencies: central retinal artery occlusion, wounds \\
\hline Severe anaemia \\
\hline Intracranial abscess \\
\hline Necrotising soft tissue infections \\
\hline Refractory osteomyelitis \\
\hline Delayed radiation injury \\
\hline Compromised grafts and flaps \\
\hline Acute thermal burn injury \\
\hline Idiopathic sudden sensorineural hearing loss \\
\hline
\end{tabular}

The use of HBOT is not without risk. ${ }^{15}$ The most common side effects of HBOT are middle ear barotrauma and sinus squeeze, which appear in almost $2 \%$ of treated patients. ${ }^{23}$ Another frequent complaint is claustrophobia, which may occur in monoplace and multiplace chambers. Multiple exposures to HBOT can also lead to oxygen toxicity. Oxygen toxicity may lead to progressive myopia and pulmonary symptoms such as cough and inspiratory pain. When higher oxygen pressures are used, especially in treatment of acidotic patients, for example, those with carbon monoxide poisoning, oxygen-induced seizures may also occur. For these reasons, a thorough medical history and physical examination should precede every HBOT. By doing this, subjects who are at risk of complications can be identified, and the right treatment protocol can be tailored to the patient. ${ }^{15}$

\section{CONCLUSION}

Migraine can be a very debilitating problem. Migraines affect approximately $15 \%$ of the general population in England and the WHO ranks migraine as the most prevalent and disabling neurological condition. Management of migraines can be broadly divided into lifestyle and trigger management, acute treatments, and preventive treatments. Despite pharmacological advances, this medical problem has remained undertreated. HBOT can be an alternative treatment for patients with migraine who are not responding to traditional pharmacotherapy. Despite the risk of using HBOT, patients at risk can be identified early before the treatment protocol; however, more studies are needed before HBOT can be a mainstream treatment of persistent migraine in the near future.

\section{References}

1. Steiner TJ et al. Migraine: the seventh disabler. J Headache Pain. 2013;14(1):1.

2. Steiner TJ et al. The prevalence and disability burden of adult migraine in England and their relationships to age, gender and ethnicity. Cephalalgia. 2003;23(7):519-27.
3. World Health Organization (WHO). The global burden of disease: 2004 update. 2004. Available at: https://www.who.int/healthinfo/ global_burden_disease/GBD_ report_2004update_full.pdf. Last accessed: January 2021.
4. Weatherall MW. The diagnosis and treatment of chronic migraine. Ther Adv Chronic Dis. 2015;6(3):115-23.

5. Dodick DW. A phase-by-phase review of migraine pathophysiology. Headache. 2018;58(Suppl 1):4-16. 
6. Cutrer FM. Pathophysiology of migraine. Semin Neurol. 2006;26(2):171-80.

7. Ashina M. Migraine. N Engl J Med. 2020;383:1866-76.

8. Charles A. Advances in the basic and clinical science of migraine. Ann Neurol. 2009;65(5):491-8.

9. Charles A. Vasodilatation out of the picture as a cause of migraine headache. Lancet Neurol. 2013;12(5):419-20.

10. Amin FM et al. Magnetic resonance angiography of intracranial and extracranial arteries in patients with spontaneous migraine without aura: a cross-sectional study. Lancet Neurol. 2013;12(5):454-61.

11. Goadsby PJ et al. Release of vasoactive peptides in the extracerebral circulation of humans and the cat during activation of the trigeminovascular system. Ann Neurol. 1988:23(2):193-6.

12. Burnstein R. Deconstructing migraine headache into peripheral and central sensitization. Pain. 2001;89(2-3): 107-10.

13. Strassman AM et al. Sensitization of meningeal sensory neurons and the origin of headaches. Nature. 1996;384(6609):560-4.

14. Kaube $\mathrm{H}$ et al. Acute migraine headache: possible sensitization of neurons in the spinal trigeminal nucleus? Neurology. 2002;58(8): 1234-8.

15. Undersea and Hyperbaric Medical Society, Moon RE (eds.), Hyperbaric Oxygen Therapy Indications (2019), $14^{\text {th }}$ edition, North Palm Beach: Best Publishing Company.

16. Thom SR. Oxidative stress is fundamental to hyperbaric oxygen therapy. J Appl Physiol (1985) 2009;106(3):988-95.

17. Memar MY et al. Hyperbaric oxygen therapy: antimicrobial mechanisms and clinical application for infections. Biomedicine and Pharmacother. 2019;109:440-7.
18. Matera DV et al. Revisiting the expanded use of hyperbaric oxygen therapy for treatment of resistant migraines. Med Gas Res. 2019;9(4):238-40.

19. Iversen HK et al. Arterial responses during migraine headache. Lancet. 1990:336(8719):837-9.

20. Niinikoski JH. Clinical hyperbaric oxygen therapy, wound perfusion and transcutaneous oximetry. World J Surg. 2004;28(3):307-11.

21. Bennet $\mathrm{MH}$ et al. Normobaric and hyperbaric oxygen therapy for migraine and cluster headache. Cochrane Database Syst Rev. 2015;12:CDO05219.

22. Thom SR. Hyperbaric oxygen - its mechanism and efficacy. Plast Reconstr Surg. 2011;127(Suppl 1):S131-41.

23. Plafki $\mathrm{C}$ et al. Complications and side effects of hyperbaric oxygen therapy. Aviat Space Environ Med. 2000:71(2):119-24 